\title{
Estágio curricular supervisionado: relato de experiência de acadêmicos de enfermagem em um hospital universitário
}

\author{
Supervised curricular internship: experience report of nursing students at a university \\ hospital
}

Pasantía curricular supervisada: informe de experiencia de estudiantes de enfermería en un hospital universitario

Mariana Paula da Silva ${ }^{1 *}$, Dhienifã Brena Marinho de Souza ${ }^{1}$, Juliana Oliveira de Lira ${ }^{1}$, Deyvylan Araujo Reis ${ }^{1}$.

\section{RESUMO}

Objetivo: Descrever as experiências vivenciadas por acadêmicos de um curso de Enfermagem do interior do Estado do Amazonas na atividade de Estágio Curricular Supervisionado (ECS) I, em um hospital universitário, localizado na capital amazonense. Relato de experiência: Trata-se de um estudo descritivo, do tipo relato de experiência, realizado durante o Estágio Curricular Supervisionado I em um hospital universitário. Neste relato, enfatizaram-se as experiências vivenciadas por discentes de enfermagem. Os alunos tiveram oportunidade de conhecer diversos setores do hospital e contribuir com eles. Entre os setores de estágio, destacam-se: Clínica Médica, Clínica Cirúrgica, Clínica Ortopédica, Clínica Neurocirúrgica, Central de Material e Esterilização, Núcleo de Segurança do Paciente, Comissão de Controle de Infecção Hospitalar (CCIH) e Ambulatório. Considerações finais: As experiências adquiridas no ECS contribuíram para o amadurecimento dos acadêmicos em diferentes cenários de atuação. Denota-se que o conhecimento se torna fundamental na formação acadêmica, pois possibilita o desenvolvimento de habilidades, liderança e tomada de decisões no mundo real de trabalho.

Palavras-chave: Estudante, Enfermagem, Estágio clínico.

\begin{abstract}
Objective: To describe the experiences lived by students of a Nursing course in the interior of the State of Amazonas in the activity of Supervised Curricular Internship (ECS) I, in a university hospital, located in the capital of Amazonas. Experience report: It is a descriptive study, of the type of experience report, carried out during the Supervised Curricular Internship I in a university hospital. In this report, the experiences of nursing students were emphasized. The students had the opportunity to get to know different sectors of the hospital and contribute to them. Among the internship sectors, the following stand out: Medical Clinic, Surgical Clinic, Orthopedic Clinic, Neurosurgical Clinic, Material and Sterilization Center, Patient Safety Center, Hospital Infection Control Commission $(\mathrm{CClH})$ and Ambulatory. Final considerations: The experiences acquired at ECS contributed to the maturation of academics in different performance scenarios. It is noted that knowledge becomes fundamental in academic training, as it enables the development of skills, leadership and decision making in the real world of work.
\end{abstract}

Keywords: Student, Nursing, Clinical internship.

1 Universidade Federal do Amazonas, Coari - AM.
${ }^{*}$ E-mail: marianapaula.mp@gmail.com

SUBMETIDO EM: $8 / 2020$

ACEITO EM: 9/2020

PUBLICADO EM: 12/2020

REAS/EJCH | Vol.12(12) | e4668 | DOI: https://doi.org/10.25248/reas.e4668.2020 Página 1 de 6 


\section{RESUMEN}

Objetivo: Describir las experiencias vividas por los estudiantes de un curso de enfermería en el interior del Estado de Amazonas en la actividad de Pasantía Curricular Supervisada (ECS) I, en un hospital universitario, ubicado en la capital de Amazonas. Informe de experiencia: Se trata de un estudio descriptivo, del tipo informe de experiencia, realizado durante la Pasantía Curricular Supervisada I en un hospital universitario. En este informe se destacaron las experiencias de los estudiantes de enfermería. Los estudiantes tuvieron la oportunidad de conocer diferentes sectores del hospital y contribuir a ellos. Entre los sectores de la pasantía se destacan: Clínica Médica, Clínica Quirúrgica, Clínica Ortopédica, Clínica Neuroquirúrgica, Centro de Material y Esterilización, Centro de Seguridad del Paciente, Comisión de Control de Infecciones Hospitalarias $(\mathrm{CCIH})$ y Ambulatorio. Consideraciones finales: Las experiencias adquiridas en el ECS contribuyeron a la maduración de los académicos en diferentes escenarios de actuación. Se observa que el conocimiento se convierte en fundamental en la formación académica, ya que permite el desarrollo de habilidades, liderazgo y toma de decisiones en el mundo real del trabajo.

Palabras clave: Estudiante, Enfermería, Prácticas clínicas.

\section{INTRODUÇÃO}

Aprimorar os conhecimentos tornou-se uma tarefa cada vez mais essencial para diversas profissões. Deste modo, o Estágio Curricular Supervisionado (ECS) é um importante recurso de ensino aplicado na Graduação em Enfermagem que almeja, mediante atividades acadêmicas, enriquecer a formação profissional (LIMA TC, et al., 2014).

De acordo com as Diretrizes Curriculares Nacionais (DCNs), o currículo de formação do enfermeiro deve dispor tanto dos objetivos teóricos e práticos próprios do curso, quanto, obrigatoriamente, o estágio supervisionado em hospitais gerais e especializados, ambulatórios, rede básica de serviços de saúde e comunidades, estes que se realizarão nos dois últimos semestres do Curso de Graduação em Enfermagem (BRASIL, 2001).

O enfermeiro desempenha diversas funções, entre elas a assistência, educação, pesquisa e gestão, todas voltadas ao cuidar humano (ALVES EATD e COGO ALP, 2014). Neste contexto, o estágio curricular demonstra ser uma ferramenta de ensino que aproxima o discente as estas funções. A inclusão desta modalidade no currículo, auxilia na construção da autonomia, liderança, resolução de conflitos e capacidade de tomada de decisão, tendo como decorrência uma formação empenhada no outro e na assistência em enfermagem (MARCHIORO D, et al., 2017). Dessa forma, o estágio é fundamental para o aperfeiçoamento de técnicas adquiridas pelos discentes durante a formação acadêmica, pois prepara o aluno para dinâmica dos serviços de saúde, atuação com a equipe de trabalho, assim como garante experiência e conhecimento para competências profissionais (RODRIGUES AMM, et al., 2014; ESTEVES LSF, et al., 2018).

Contudo, além das habilidades técnicas que são aperfeiçoadas durante a prática curricular, o aluno também pode ampliar o pensamento crítico e reflexivo que consequentemente contribuirá para 0 entendimento do processo de saúde-doença, a fim garantir aos usuários melhor qualidade nos serviços de saúde (RAMOS TK, et al., 2018). Diante do exposto, a experiência do estágio curricular possui grande relevância para a formação acadêmica por ser uma atividade indispensável na formação dos sujeitos envolvidos. Este estudo teve como objetivo descrever as experiências vivenciadas por acadêmicos do curso de Enfermagem de uma Instituição de Ensino Superior Pública na atividade de Estágio Curricular Supervisionado I realizado em um Hospital Universitário.

\section{RELATO DE EXPERIÊNCIA}

Este estudo fundamenta-se no relato das experiências vivenciadas no Estágio Supervisionado que ocorreu em um Hospital Universitário. O método empregado consistiu na descrição da disciplina, observação, campo de estágio e as atividades realizadas. 
O ECS apresenta-se como uma disciplina ofertada no segundo semestre de 2019, sendo composta por três docentes que também são preceptores. Para organização, a coordenação do hospital juntamente com os docentes responsáveis já havia pré-estabelecido as unidades para receber os alunos. Todos eles ficaram sob a responsabilidade dos docentes do ECS e de supervisores/enfermeiros atuantes no hospital.

No mês de outubro de 2019, foram realizadas reuniões com os integrantes do ECS (estudantes, orientadores e supervisores). Foram apresentadas plano de curso, atribuições dos discentes, cronograma de atividade, instrumento de avaliação, planilhas com temas de educação em saúde, termo de compromisso para realização do estágio e roteiro de acompanhamento para os supervisores de cada unidade do hospital.

Após as orientações, houve uma visita prévia ao Hospital Universitário com o intuito de integrar ensinoserviço. Os discentes conheceram Clínica Médica, Clínica Cirúrgica, Clínica Ortopédica, Clínica Neurocirúrgica, Central de Materiais e Esterilização (CME), Núcleo de Segurança do Paciente (NSP), Comissão de Controle de Infecção Hospitalar $(\mathrm{CClH})$ e Ambulatório.

A coordenação de enfermagem do hospital recepcionou os universitários para realizar a integração. $O$ processo deve ser realizado junto aos estagiários para apresentação das normas e rotinas que devem ser seguidas durante o estágio. Deste modo, os acadêmicos foram inseridos em atividades práticas de competência do profissional enfermeiro.

Realizaram-se atividades nas clínicas médica, cirúrgica, ortopédica e neurocirúrgica, aplicando a assistência de enfermagem principalmente a pacientes internados com complicações clínicas provenientes de processos cirúrgicos ou de natureza aguda ou causados por alguma comorbidade, além do planejamento e intervenção coerentes com a sistematização da assistência de enfermagem.

No Hospital Universitário, a rotina na clínica médica inicia-se com a passagem do plantão entre os enfermeiros responsáveis. Após isso, é feita a visita de enfermagem e a inspeção geral do estado do paciente com uso de instrumentos contendo informações que são necessárias para classificá-lo para o nível de assistência ideal. Além disso, foram realizadas admissão dos pacientes, administração de subcutâneos, curativos complexos, avaliação do risco de queda e lesão por pressão, orientações ao paciente e acompanhante, implementação da sistematização, passagem de sonda vesical, acesso por hipodermóclise, entre outras.

Quanto à clínica cirúrgica, a assistência de enfermagem é centrada nos cuidados pré e pós-operatórios, ofertada a clientes provenientes de diferentes especialidades. No pré-operatório, houve o preparo de pacientes para cirurgias e orientações adicionais para minimizar a ansiedade e preocupação. Os acadêmicos também realizaram cuidados no pós-operatório, como monitorar sinais vitais, as funções fisiológicas e o local da cirurgia a fim de prevenir infecções.

O Hospital Universitário também possui as clínicas ortopédica e neurocirúrgica. A primeira atende pacientes com afecções ortopédicas e traumatológicas, e a enfermagem. Centrou-se os cuidados no tratamento clínico ou cirúrgico desses clientes, prestando a assistência e desenvolvendo ações de prevenção e promoção da saúde.

A clínica neurocirúrgica é preparada para atender patologias relacionadas ao Sistema Nervoso Central (SNC) e pacientes de outras especialidades, tais como oftalmologia e otorrinolaringologia. A assistência de enfermagem neste setor, também é voltada para os cuidados no pré e pós-operatório. Enfatiza-se, os cuidados realizados no pós-operatório, pois os curativos em sua maioria possuíam drenos e eram de grande extensão.

A Central de Materiais Esterilização (CME) também fez parte do campo de estágio dos acadêmicos de enfermagem. Os discentes verificaram a temperatura dos ambientes da CME por meio dos termômetros instalados em locais estratégicos, sendo essa a primeira tarefa do enfermeiro no setor; inspeção de todos os materiais recebidos no plantão, bem como as suas condições, balanço dos materiais disponíveis em estoque e levantamento dos que seriam necessários solicitar pelo aplicativo do hospital. 
Além da assistência de enfermagem oferecida a pacientes nas clínicas, os discentes também realizaram atividades administrativas, principalmente nos setores como o Núcleo de Segurança do Paciente (NSP), Comissão de Controle de Infecção Hospitalar $(\mathrm{CClH})$ e o ambulatório. Diferentemente das clínicas, onde os discentes tiveram um papel assistencial, as demandas desse setor eram administrativas, pois as atividades realizadas eram com os prontuários, documentos de pacientes e protocolos do departamento e raramente tendo contato com pacientes.

O NSP possui diversas demandas, entre elas, buscas ativas em clínicas. Ela é feita para averiguar a utilização das pulseiras de identificação e prontuários dos pacientes. Desse modo, eram conferidos dados básicos, medida importante para evitar que procedimentos sejam realizados em pacientes de forma equivocada. Se houvessem pacientes sem a pulseira, era realizada a notificação como rotina exigida. As notificações podem ser feitas por qualquer profissional no sistema próprio do hospital.

O hospital possui também a Comissão de Controle de Infecção Hospitalar (CCIH), que tem como gerente um profissional enfermeiro. Antes de iniciarem as atividades práticas, os discentes realizaram leituras minuciosas para entendimento das responsabilidades atribuídas no setor, que consequentemente colaboravam com a equipe na elaboração e avaliação das ações de prevenção e controle das infecções. As buscas ativas na $\mathrm{CClH}$ também eram frequentes, analisavam-se prontuários para identificar casos de infecções. Além disso, também realizaram a busca fornada, que é um instrumento utilizado para investigar se houve casos de pacientes com infecção hospitalar, sobretudo, de sítio cirúrgico, após a saída destes do hospital.

O Ambulatório do hospital também oferece serviços assistenciais. O local também dispõe de diversas unidades assistenciais, tais como: dermatologia, reumatologia, ginecologia, oftalmologia, pneumologia, cardiologia, ortopedia, entre outras especialidades. E, para gerenciá-lo, conta com uma enfermeira, a qual apresentou aos discente o funcionamento, a organização e a estrutura física, explanando de maneira detalhada as atividades que cada unidade realizava.

As atividades realizadas no Ambulatório, foram principalmente gerenciais. Os acadêmicos acompanharam a enfermeira do setor executando, sobretudo, as atribuições como: reagendamento de consultas, atualização do livro de ocorrências, solicitação de materiais e insumos, elaboração de planilhas e escalas dos profissionais, trocas de plantão e agendamento de férias. Dessa forma, nota-se a importância do desenvolvimento desta competência e habilidade no ECS, pois, ao longo da graduação, não é diretamente oportunizado ao discente a realização de atividades gerenciais.

\section{DISCUSSÃO}

A recepção do acadêmico é importante para o processo de adaptação ao setor de estágio. O contato com a equipe necessita ser feito após o contato com o docente responsável, o que permite garantir a participação do enfermeiro do setor (RESTELATTO MTR e DALLACOSTA FM, 2018). Este momento é relevante, pois a forma como o enfermeiro realiza suas atividades dará referência para sua equipe e consequentemente habilidades e competências aos acadêmicos (SOUZA RV, et al., 2017).

A presença de docentes no cenário de prática também contribui para uma aprendizagem eficaz, pois atuam de forma intermediária em possíveis conflitos, garantem a comunicação durante o estágio e orientam nas tomadas de decisões e atividades a serem desenvolvidas pelos discentes intervindo quando necessário (MARCHIORO D, et al.,2017; MARRAN AL, et al., 2015; BEGNATO MHS, 2015).

As experiências adquiridas durante o estágio, colaboram para o acadêmico descobrir habilidades profissionais de assumir responsabilidades, que são designadas durante a prática e se tornarão essenciais a sua futura formação (EVANGELISTA DL e IVO OP, 2014). Contudo, durante a graduação, o acadêmico se depara com as aulas práticas contendo procedimentos de Enfermagem que não se associam a forma sistematizada de serviços (RAMOS TK et al.,2018). O processo de formação dos estudantes de enfermagem não deve ser restrito a forma técnica e instrumental, mas na construção do pensamento crítico-reflexivo com objetivo de somar nas atribuições do trabalho de enfermagem (NUNES NJS, et al., 2016). 
Desta forma, o ECS oportuniza a atuação do acadêmico em diferentes cenários de práticas como colaborar para o incremento das ações profissionais e auxiliar no desenvolvimento de valores éticos, morais que norteiam a sua profissão (ESTEVES LSF, et.,2018).

Os estágios curriculares, têm demonstrado ser para os estudantes de diversas áreas, o lugar ideal para o desenvolvimento de habilidade baseada em conhecimentos científicos e que propiciarão o contato direto com o mercado de trabalho (RIGOBELLO JL, et al., 2018). Considerando que a aprendizagem e o preparo exigido dos profissionais da saúde sejam contínuos, o contato com pacientes durante o ECS é essencial para 0 desenvolvimento das habilidades de assistência ao paciente.

Em um estudo realizado com alunos do último semestre da graduação em Enfermagem de uma Universidade Federal, adotando o Questionário de Competências Clinicas (QCC) demonstrou-se que após a realização do estágio curricular houve aperfeiçoamento de técnicas e habilidades no ambiente hospitalar (ROCHA ENDA, et al., 2019).

Neste contexto, a prática em campo hospitalar propicia ao discente o contato diário das situações reais da sua profissão. A utilização da Sistematização da Assistência de Enfermagem (SAE) como ferramenta de trabalho, também tem demonstrado ser relevante para a atuação, pois ela tem o intuito de identificar as necessidades dos pacientes e propor cuidados necessários que deverão ser realizados pela equipe (NEGREIROS RV e LIMA VCB, 2018).

Ressalta-se que, o ensino clínico da enfermagem através dos estágios, tem o objetivo de oferecer ao acadêmico conhecimentos inerentes a profissão que permitirão aperfeiçoar habilidades. Durante essas práticas, é possível estabelecer maior contato com pacientes, colegas e docentes contribuindo para uma atuação crítica e reflexiva (NETO AV, et al., 2018; LIMA JÁ, et al., 2016). De acordo com Leal LA, et al. (2017), os cuidados com pacientes e os conhecimentos teórico-práticos são competências indispensáveis para a assistência hospitalar.

Observa-se que a vivência no ECS colabora para o contato com as diversas disposições do profissional enfermeiro, inclusive gerenciais. As competências gerenciais presentes na DCN: tomada de decisão, comunicação, liderança administração e gerenciamento são essenciais durante as práticas curriculares (BRASIL, 2001).

No gerenciamento de enfermagem, a tomada de decisão tem o objetivo de sistematizar e deliberar condutas adequadas de acordo com evidencias cientificas; a comunicação serve como forma de interação com profissionais e público geral; liderança conduz o trabalho em equipe de forma organizada e responsável. Ressalta-se, que estas competências darão subsídio para a gestão de recursos humanos, materiais, físicos e financeiros que são atividades comuns no dia-a-dia do enfermeiro (SADE PMC, et al., 2014).

Durante o processo de formação, o acadêmico necessita desenvolver competências de gestão de forma a abranger o gerenciamento de enfermagem. Espera-se também, que o aluno desenvolva um perfil de liderança ideal para minimizar conflitos durante a assistência colaborando para a qualidade dos serviços assistenciais (TEIXEIRA NL, et al., 2018).

Um estudo realizado com enfermeiros na atenção básica de saúde, a liderança foi expressa como atributo imprescindível para a formação, sobretudo para a graduação de enfermagem (LANZONI, GMM, et al., 2016). Nesse contexto de atuação do enfermeiro, é possível perceber que a gerência e a assistência de enfermagem integram as responsabilidades de sua competência que, consequentemente, contribuem para organização e qualidade nos serviços de saúde ofertados a pacientes e familiares que buscam intervenções no processo saúde-doença (SOARES MI, et al., 2015; MATTOS JCO e BALSANELLI AP, 2019). As funções administrativas exercidas pelo enfermeiro têm se demonstrado como prática essencial. Além das responsabilidades relacionadas aos pacientes, cabe ao enfermeiro gerenciar setores, serviços e a equipe de enfermagem (TREVISAN DD, et al., 2013).

Contudo, o gerenciamento de enfermagem envolve não só a organização do serviço de saúde, mas também da equipe que nele atua. A comunicação, liderança e tomada de decisão são essenciais para que 0 enfermeiro realize uma gestão eficaz. Ressalta-se que, através da gerência, é possível adquirir experiências que darão subsídio para uma prática administrativa eficiente (BENITO GAV, et al., 2012). 
Assim, foram observadas durante o ECS diversas atividades que compõem a formação do discente. As oportunidades vivenciadas em diferentes contextos de atuação influenciam o processo de formação, haja vista que os acadêmicos tiveram experiências únicas que Ihes possibilitarão maior segurança e conhecimento nas práticas profissionais futuras (LIMA TC, et al., 2014).

As experiências adquiridas nos estágios supervisionados contribuem para o amadurecimento dos acadêmicos por meio das vivências em diferentes cenários de atuação. Destarte, os conhecimentos adquiridos tornam-se fundamentais na formação acadêmica, pois possibilitam a ampliação de habilidades, raciocínio crítico, liderança e tomada de decisão no mundo real do trabalho.

Além disso, essa aquisição é um passo importante para a caminhada do recém-formado que se lança ao mundo de trabalho. Dessa forma, embora sejam reais e explícitos os conflitos vivenciados pelo estudante, durante o período de ECS, nota-se que, ao adentrar o cotidiano do Enfermeiro, há uma progressão para aquisição de experiências que tendem a fortalecê-lo no processo de formação, tendo em vista que a transposição da realidade do acadêmico pode ser muitas vezes uma fase difícil, pois envolve adaptações e também superação nos futuros ambientes de trabalho.

\section{AGRADECIMENTOS}

A todos os profissionais do Hospital Universitário por todo apoio e por proporcionarem um ambiente propício para o ganho de experiências e conhecimento. A direção e corpo docente da Universidade Federal que contribuíram para que o estágio ocorresse. Em especial, aos nossos preceptores que não mediram esforços para nos acompanharem durante o período de estágio.

\section{REFERÊNCIAS}

1. ALVES EATD, COGO ALP. Percepção de estudantes de enfermagem sobre o processo de aprendizagem em ambiente hospitalar. Rev Gaúcha Enferm, 2014;35 (1):102-109, 2014

2. BENITO GAV, et al. Desenvolvimento de competências gerais durante o estágio supervisionado. Rev Bras Enferm, 2012; 65(1):1728.

3. BRASIL. Ministério da Educação. Conselho Nacional de Educação. Resolução n 3, de 7 de novembro de 2001 . Institui Diretrizes Curriculares Nacionais do Curso de Graduação em Enfermagem. 2001. 4. ESTEVES LSF, et al. O estágio curricular supervisionado na graduação em enfermagem: revisão integrativa. Rev Bras Enferm,

5. EVANGELISTA DL, IVO OP. Contribuições do estágio supervisionado para a formação do profissional de enfermagem: expectativas e desafios. Rev Enferm Contemp, 2014; 3(2):123-30.

6. LANZONI GMM, et al. Práticas de liderança do enfermeiro na atenção básica à saúde: uma teoria fundamentada nos dados. Texto \& Contexto Enferm, 2016;25(4):2-9.

7. LEAL LA, et al. Competências clínicas e gerenciais para enfermeiros hospitalares: visão de egressos de enfermagem. Rev Bras Enferm, 2018; 71(suppl 4):1605-12.

8. LIMATC, et al. Estágio curricular supervisionado: análise da experiência discente. Rev Bras Enferm. 2014; 67(1): 133-40.

9. LIMA JÁ, et al. Expectativas do estágio hospitalar para estudantes de enfermagem. Revista Brasileira Ciências da Saúde, 2016 ; 14 (48): 5-10.

10. MARRAN AL, et al. As políticas educacionais e o estágio curricular supervisionado no curso de graduação em enfermagem. Trab. educ. saúde, 2015;3(1): 89-108.

11. MARCHIORO D, et al. Estágio curricular supervisionado: relato dos desafios encontrados pelos (as) estudantes. Arq Ciênc Saúde UNIPAR, 2017; 21(2):119-22.

12. MATTOS JCO, BALSANELLI APA. LIDERANÇA DO ENFERMEIRO NA ATENÇÃO PRIMÁRIA À SAÚDE. Revista Enfermagem em foco, 2019; $10(4): 164-171$

13. NEGREIROS R.V, LIMA VC. Importância do estágio supervisionado para o Acadêmico de Enfermagem no Hospital: Compartilhando experiências vivenciadas com a equipe de Trabalho. Revista da Universidade Vale do Rio Verde, 2018; $16(2): 1-7$.

14. NETO AV, et al. Sentimentos e percepção do estudante de enfermagem sobre o acolhimento no estágio. Revista Interdisciplinar,2018; 11(2): 28-36.

15. RAMOS TK, et al. Estágio curricular supervisionado e a formação do enfermeiro: atividades desenvolvidas. Rev EnfermUFSM,2018; $8(1): 59-71$.

16. RESTELATTO MTR, DALLACOSTA FM. Vivências do acadêmico de enfermagem durante o estágio com supervisão indireta. Enferm. Foco, 2018; 9 (4): 34-38.

17. RIGOBELLO JL, et al. Ações assistenciais e gerenciais desenvolvidas no estágio curricular supervisionado: impressão dos atores envolvidos. Rev EscEnferm USP, 2018; 52:1-9.

18. RODRIGUES AMM, et al. Preceptoria na perspectiva da integralidade: conversando com enfermeiros. Revista gaúcha de enfermagem / EENFUFRGS, 2014; 35(2): 106-112.

19. ROCHA ENDA, et al. Nursing Students' Perception of Clinical Competences. Reme Revista Mineira de Enfermagem, 2019; 23:e1179.

20. SADE PMC, et al. Formação Das Competências Gerenciais Do Enfermeiro: Revisão Integrativa. Ver enferm UFPE on line., Recife, 2014;8(6):1739-45.

21. SOUZA RV, et al. Imagem do Enfermeiro sob a Ótica do Acadêmico de Enfermagem. Enferm. Foco, $2017 ; 8(1)$ : 47-51.

22. SOARES MI, et al. Sistematização da Assistência de Enfermagem: facilidades e desafios do enfermeiro na gerência da assistência. Esc Anna Nery RevEnferm, 2015; 19(1):43-57.

23. TEIXEIRA NL, et al. Desafios do enfermeiro no gerenciamento de conflitos dentro da equipe de enfermagem. Rev. Adm. Saúde, $2018 ; 18(73)$.

24. TREVISAN DD, et al. Formação de enfermeiros: distanciamento entre a graduação e a prática profissional. CiêncCuid Saúde, 2013;12(2):331-7. 